\title{
UM CONJUNTO DE PLANILHAS ELETRÔNICAS PARA IDENTIFICAÇÃo E QUANTIFICAÇÃO DE CONSTITUINTES DE ÓLEOS ESSENCIAIS
}

\author{
Humberto R. Bizzo ${ }^{\mathrm{a}, *,(1)}$, Eduardo G. Barboza ${ }^{\mathrm{b}}$, Marcelly C. S. Santos ${ }^{\mathrm{a}}$ e Paola E. Gama \\ aEmbrapa Agroindústria de Alimentos, Avenida das Américas 29501, 23020-470 Rio de Janeiro - RJ, Brasil \\ bEscola de Química, Universidade Federal do Rio de Janeiro 21941-909 Rio de Janeiro - RJ, Brasil
}

Recebido em 19/06/2019; aceito 26/09/2019; publicado na web em 08/01/2020

\begin{abstract}
A SET OF ELECTRONIC SHEETS FOR THE IDENTIFICATION AND QUANTIFICATION OF CONSTITUENTS OF ESSENTIAL OILS. The identification of essential oils constituents has been made easier and faster by the use of automatic software search engines that compare the experimental mass spectra and calculate the retention indices with those in commercial databases. However, these search engines and databases are very expensive. Electronic sheets have been assembled in a set of linked sheets, so that after some data is inserted, linear retention indices, relative response factors, normalization, area correction and absolute quantitation are calculated automatically. The set has been applied to the analysis of essential oils and volatiles in laboratory routine and substantial reduction in time-consuming and error-prone repetitive work were observed. One specific example for the analysis of an essential oil is provided. The designed electronic sheets can be a costless alternative to commercial software for data handling and identification.
\end{abstract}

Keywords: essential oil analysis; gas chromatography; mass spectrometry; linear retention indices; automatation.

\section{INTRODUÇÃO}

Óleos essenciais (OE) são definidos como os produtos obtidos a partir de matérias-primas naturais de origem vegetal por destilação (arraste) a vapor, por destilação a seco ou pelo uso de processos mecânicos (prensagem) para o epicarpo de frutos cítricos, após a separação da fase aquosa (se houver), por processos físicos. ${ }^{1}$ Diversos outros extratos naturais, obtidos com o uso de solventes, fluidos supercríticos ou empregando técnicas de headspace, ainda que não sejam classificados como óleos essenciais, apresentam substâncias voláteis em sua composição e sua análise segue, via de regra, protocolos semelhantes aos utilizados para os óleos essenciais.

Embora haja alguns óleos essenciais de constituição química bastante simples, com poucos componentes, ${ }^{2}$ em sua maioria são constituídos por várias dezenas ou algumas centenas de constituintes, como no caso do OE de vetiver. ${ }^{3}$

Para identificação e quantificação, os componentes dos OE precisam ser separados antes ou durante o processo analítico. Com a introdução da cromatografia em fase gasosa de alta resolução verificou-se um grande avanço na análise dos constituintes dos óleos essenciais. ${ }^{4}$

Na era pré-cromatografia gasosa e mesmo nos seus primórdios, para a identificação (análise qualitativa) era necessário isolar cada constituinte, em cromatografia preparativa, para a realização de estudos de degradação, preparação de derivados ou espectroscópicos (IV, RMN e EM).

Uma vez separados, os componentes podiam ser quantificados utilizando-se, preferencialmente, um detector de ionização de chama ou de condutividade térmica. Em função da composição complexa dos $\mathrm{OE}$, bem como dos efeitos sobre o perfil olfativo, os maiores esforços e atenções foram concentrados na análise qualitativa, isto é, na identificação dos constituintes, e a sua quantificação foi considerada menos importante. ${ }^{5}$

$\mathrm{O}$ uso dos índices de retenção, originalmente proposto por Kováts, ${ }^{6}$ para sistemas isotérmicos (IK), e adaptado por Van den Dool e Kratz, ${ }^{7}$ para análises com programação linear de temperatura (IRL),

*e-mail: humberto.bizzo@embrapa.br facilitaram o processo de identificação. Numa época, entretanto, onde um grande número de fases estacionárias comerciais estava disponível e, além disso, outras tantas eram preparadas nos próprios laboratórios nos quais a pesquisa era conduzida, o uso de índices de retenção ainda era dependente da existência de padrões autênticos ou do isolamento, para a determinação do índice de retenção original de cada componente.

A partir da década de 1960 passou-se a usar um detector de massas acoplado à saída do cromatógrafo, o que poderia dispensar a necessidade de isolamento para a identificação de novas substâncias. Apenas o espectro de massas, entretanto, não é suficiente para a identificação inequívoca dos constituintes dos $\mathrm{OE}$, já que muitos deles são isóbaros e/ou isômeros entre si e, consequentemente, se fragmentam de forma semelhante, produzindo espectros de massas similares. ${ }^{8}$

A combinação das técnicas de espectrometria de massas e dos índices de retenção permitiu a identificação de uma quantidade razoável de componentes. Diversas coleções combinadas de dados, com espectros de massas e índices de retenção tornaram-se disponíveis. Provavelmente, a coleção mais utilizada é aquela do livro do professor Robert Adams. ${ }^{9}$ Hoje, bases acessíveis via internet, gratuitas, já estão disponíveis, tais como Webbook, ${ }^{10}$ Flavornet, ${ }^{11}$ e Pherobase.${ }^{12}$

Em análise de rotina para componentes-alvo (target analysis), como pesticidas, fármacos, derivados de petróleo e outros, os próprios softwares de controle dos cromatógrafos permitem a realização da análise quantitativa, a partir de dados de curvas analíticas, fatores de resposta e seleção de quais componentes devem ser quantificados, a partir de janelas de tempo de retenção definidas. Até os nomes dos componentes podem ser listados no cromatograma ou no relatório de dados, desde que uma identificação prévia tenha sido feita e os tempos de retenção não variem. ${ }^{13}$

Entretanto, no caso de óleos essenciais e outros produtos naturais, principalmente nas atividades de pesquisa, os componentes presentes não são previamente conhecidos. ${ }^{8}$ Mais ainda, dada a complexidade de algumas amostras, nas quais observa-se coeluição parcial ou mesmo total, essa ferramenta disponível nos softwares de controle dos equipamentos não é eficaz. Não é raro encontrar, em duas amostras distintas, componentes diferentes no mesmo tempo de eluição, 
como ocorre com limoneno e beta-felandreno, por exemplo, quando analisados em uma coluna com fase de metilsilicone.

O problema passou a ser o processamento da grande quantidade de dados gerada nas análises. Embora processos de automação de busca e seleção de informações já tivessem sido desenvolvidos, ${ }^{14}$ foi somente com a introdução dos microcomputadores nos anos 1990 junto aos sistemas de cromatografia em fase gasosa acoplada à espectrometria de massas que se tornou possível o processamento dos dados de forma veloz, tanto para a comparação dos espectros de massas experimentais com os da espectroteca quanto o cálculo e comparação dos índices de retenção ou, ainda, as duas operações combinadas. Todo este processamento é realizado a partir dos dados brutos do cromatograma de íons totais, de forma automatizada, e listas de possíveis identificações são, então, apresentadas aos usuários. Há diversas espectrotecas especializadas em diferentes classes de substâncias e matrizes, ${ }^{15}$ inclusive bases de dados de óleos essenciais. ${ }^{16}$ Entretanto, seu custo é muito elevado, variando de US\$2,775 a US\$10,000, ${ }^{17}$ valores que podem estar fora do orçamento de muitos laboratórios tanto no momento da compra do sistema cromatográfico quanto para aquisição posterior.

Planilhas de cálculo usando funções pré-existentes no software Microsoft Excel ${ }^{\circledR}$ foram desenvolvidas para as diferentes etapas do processo de identificação e quantificação. Posteriormente, foram reunidas e interconectadas, de modo a se reduzir o tempo consumido no processo, bem como minimizar erros de transcrição de dados. Nesta nota técnica são apresentadas as etapas de desenvolvimento e uso do conjunto de planilhas.

\section{PARTE EXPERIMENTAL}

\section{Preparo da amostra}

\section{Padrão interno}

Um padrão interno (PI) deve ser adicionado à amostra para a quantificação. Uma solução prévia do padrão interno deve ser preparada com o mesmo solvente a ser utilizado na diluição da amostra. Foram testadas separadamente duas substâncias: octanoato de metila 99\% e octadecano 99\% (Sigma-Aldrich, São Paulo).

\section{Padrões para o cálculo dos fatores de resposta (FR)}

As substâncias representativas usadas para o cálculo dos fatores de resposta foram: $\alpha$-pineno, $\gamma$-terpineno e $p$-cimeno para os monoterpenos; linalol, nerol, mentol, carvona e óxido de linalol para os monoterpenoides oxigenados; $(E)$-cariofileno (sesquiterpenos); $\alpha$-bisabolol (sesquiterpenoides oxigenados); acetato de mentila e acetato de geranila (ésteres). Todos os padrões são comerciais e foram fornecidos pela Sigma-Aldrich (Milwaukee, USA), com pureza mínima de $98 \%$. Deve-se utilizar ao menos um padrão para cada classe química identificada no óleo essencial ou extrato volátil. Foram preparadas soluções em 5 concentrações diferentes para cada padrão utilizado $\left(0,1 \mathrm{mg} \mathrm{mL}^{-1}, 0,2 \mathrm{mg} \mathrm{mL}^{-1}, 0,4 \mathrm{mg} \mathrm{mL}^{-1}, 0,8 \mathrm{mg} \mathrm{mL}^{-1} \mathrm{e}\right.$ $\left.1,6 \mathrm{mg} \mathrm{mL}^{-1}\right)$, que foram injetadas em triplicata. Os fatores de resposta relativos ao octadecano $\left(0,1 \mathrm{mg} \mathrm{mL}^{-1}\right)$ foram calculados segundo a equação (2), inserida na Planilha 6.

\section{Pesagem e diluição da amostra}

Exatamente cerca de $100 \mathrm{mg}$ de um óleo essencial (ou outro tipo extrato) a ser analisado foram pesados diretamente em balão volumétrico de $10 \mathrm{~mL}$. Foram adicionados cerca de $5 \mathrm{~mL}$ de solvente. $\mathrm{Na}$ rotina do laboratório usa-se hexano para diluir os óleos essenciais. Diclorometano (ou outro solvente adequado) também podem ser utilizado. Exatamente $10,0 \mu \mathrm{L}$ de uma solução a $1 \%$ do PI foram adicionados com o auxílio de uma microseringa Hamilton (Hamilton Company, Reno-EUA) e o volume final do balão ajustado a $10 \mathrm{~mL}$.
Cerca de $2 \mathrm{~mL}$ foram transferidos para um frasco próprio para uso em injetor automático e o frasco foi selado. Outras quantidades podem ser usadas, pois a planilha calcula as quantidades e diluições efetivamente utilizadas a cada análise.

\section{Cromatografia em fase gasosa e espectrometria de massas}

A análise por cromatografia em fase gasosa foi realizada em um cromatógrafo Agilent (Wilmington, DE) modelo 7890A, equipado com um injetor automático 7693 e um detector de ionização por chama (DIC). Utilizou-se uma coluna capilar de silica fundida DB-5MS (5\%-fenil-95\% metilsilicone, $30 \mathrm{~m}$ x 0,25 mm x 0,25 $\mu \mathrm{m}$ ) e hidrogênio

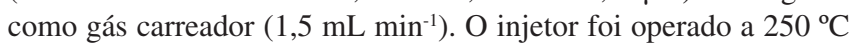
com divisão de fluxo de 1:50. A temperatura da coluna capilar foi variada de $60{ }^{\circ} \mathrm{C}$ a $240{ }^{\circ} \mathrm{C}$, a uma taxa de $3,0{ }^{\circ} \mathrm{C} \mathrm{min}{ }^{-1} .{ }^{9} \mathrm{O}$ volume de amostra injetado foi de $1,0 \mu \mathrm{L}$ da solução preparada no item anterior. Para a quantificação, os dados de área absoluta gerados pelo DIC foram transferidos diretamente para a Planilha 1.

Os espectros de massas foram obtidos com um cromatógrafo Agilent (Wilmington, DE) modelo 6890, equipado com um injetor automático 7693 e acoplado a um detector seletivo de massas Agilent $5973 \mathrm{~N}$. O mesmo volume de amostra $(1,0 \mu \mathrm{L})$ foi injetado e a mesma coluna cromatográfica descrita acima foi utilizada, porém hélio como gás carreador (1,0 $\left.\mathrm{mL} \mathrm{min}^{-1}\right)$. A programação de temperatura do forno, a temperatura do injetor e a taxa de divisão de fluxo foram as mesmas usadas para a cromatografia em fase gasosa. O detetor de massas foi operado em modo de varredura (scan) e ionização eletrônica, a 70 eV. Os espectros de massa experimentais foram comparados com dados das bibliotecas Adams, ${ }^{9}$ Wiley ${ }^{15}$ e NIST. ${ }^{18}$

\section{Planilhas}

O conjunto de planilhas foi desenvolvido em Excel $^{\circledR} 2013$ utilizando fórmulas pré-existentes no programa. No Material Suplementar são listadas as fórmulas usadas em cada planilha e o link para um arquivo Excel com um modelo para o conjunto de planilhas. Aqui serão descritos os procedimentos para a introdução de dados e a função de cada planilha.

\section{Planilha 1: Dados das amostras}

Nesta planilha foram inseridos os dados cromatográficos brutos, ou seja, o tempo de retenção e a área absoluta de cada pico. Os dados podem ser colados diretamente a partir da tabela do software de controle do cromatógrafo. A massa de cada amostra pesada também deve ser informada em célula previamente definida. Replicatas podem ser adicionadas na mesma planilha. O exemplo contido no Material Suplementar foi elaborado para análises em triplicata.

\section{Planilha 2: Tempos de retenção (dos constituintes da série homóloga)}

Os tempos de retenção dos constituintes da série homóloga utilizada no cálculo dos índices de retenção lineares são digitados em ordem crescente e, na tabela ao lado, o correspondente número de átomos de carbono de cada padrão. Nesse trabalho usou-se uma série de $n$-alcanos variando do heptano ao hexacosano $\left(\mathrm{C}_{7}-\mathrm{C}_{26}\right)$. Os dados dessa planilha são a base para o cálculo dos IRL.

\section{Planilha 3: Dados do PI}

Inserir o conjunto de informações relativo ao padrão interno, incluindo fabricante, teor de pureza, massa de PI pesada, volume de diluição, quantidade adicionada à amostra e volume de diluição da amostra. A planilha calcula a massa de padrão efetivamente adicionada à amostra. 


\section{Planilha 4: Cálculo dos índices de retenção lineares}

Planilha na qual foram calculados os índices de retenção. Não há dados a adicionar aqui. Para automatizar a interpolação dos tempos de retenção $\left(t_{R}\right)$ de cada componente de uma amostra como os $t_{R}$ dos padrões da série homóloga, isto é, aqueles que eluem antes e depois de cada componente, foi utilizada a função "PROCV" do Excel ${ }^{\circledR}$. Para o $t_{R}$ do padrão que elui imediatamente após o pico de interesse, utilizou-se uma combinação das funções "ÍNDICE" e "CORRESP" ${ }^{19}$ Finalmente, o índice de retenção linear do pico é calculado utilizando a aproximação de Van den Dool e Kratz, ${ }^{7}$ conforme a equação (1) a seguir.

$$
I R L=100 . n \frac{\left(t_{R} x-t_{R} C n\right)}{\left(t_{R} C n+1-t_{R} C n\right)}
$$

onde: $\mathrm{t}_{\mathrm{R}} x$ é o tempo de retenção de um componente " $\mathrm{x}$ " qualquer; $\mathrm{t}_{\mathrm{R}} C n$ é o tempo de retenção do padrão " $n$ " que elui antes de " $\mathrm{x}$ "; $\mathrm{t}_{\mathrm{R}} C n+1$ é o tempo de retenção do padrão " $n+1$ ", ou seja, que elui após "x"; " $n$ " é número de átomos de carbono do padrão que elui antes de " $\mathrm{x}$ ".

\section{Planilha 5: Qualitativa}

Após comparação visual dos espectros de massas experimentais e os da biblioteca (digital ou não), bem como comparação dos índices de retenção calculados com aqueles da literatura, são digitadas as identidades (nomes) dos constituintes e os respectivos IRL da literatura consultada. Identifica-se, então, a classe química a que pertence cada componente. Essa informação será usada, na planilha 6, para se atribuir um fator de resposta (FR) para o constituinte e para o cálculo do fator de resposta relativo (FRR), que será usado nos cálculos para quantificação.

\section{Planilha 6: Fator de resposta relativo (FRR)}

O FRR é calculado nesta planilha a partir do FR de cada componente identificado na planilha "Qualitativa" e o FR do PI. Não há inserção direta de dados nesta planilha. Para o cálculo dos fatores de resposta, foram injetados ao menos um padrão (mais de um, sempre que disponível) de uma classe química (5 repetições de cada), tendo-se obtido o FR por média simples dos resultados. ${ }^{19,20}$ Este valor foi corrigido pelo FR do padrão interno, obtendo-se o FRR conforme descrito em (2)

$$
F R R=\frac{C p n}{A p n} \times \frac{C p i}{A p i}
$$

onde: Cpn é a concentração (ou massa) do padrão autêntico "n" (um monoterpeno, por exemplo); Cpi é a concentração (ou massa) do padrão interno; Apn é a área absoluta do padrão autêntico "n"; Api é a área absoluta do padrão interno.

\section{Planilha 7: Quantitativa}

Nesta planilha são efetuados os cálculos de quantificação. Inicialmente as áreas absolutas, informadas na Planilha 1, são normalizadas com o padrão interno, corrigidas com o fator de resposta relativo e os resultados são tabulados na forma de quantificação relativa (percentual) e também quantificação absoluta.

\section{Planilhas 8-11: Resultados}

São planilhas de saída (outputs) com os resultados da análise nas diferentes apresentações possíveis. Para concluir a atividade, elaborar um laudo ou outra forma de registro, basta copiar e colar a tabela de resultado mais conveniente.

\section{RESULTADOS E DISCUSSÃO}

Um exemplo do uso do conjunto de planilhas é apresentado a seguir, com dados de uma análise de rotina do óleo essencial de Lippia alba (Verbenaceae) amostra RFV 2556. O conjunto de planilhas também foi usado na análise dos constituintes voláteis de flores de Temnadenia odorifera (Apocynaceae) amostrados por headspace dinâmico ${ }^{20} \mathrm{e}$ do óleo essencial destilado de folhas de Bidens graveolens (Asteraceae). ${ }^{21}$

\section{Análise qualitativa}

Inicialmente, os dados brutos (tempos de retenção e áreas) da análise cromatográfica são inseridos diretamente na Planilha 1 (Dados da Amostra), exemplificado na figura 1. Neste exemplo, a mesma amostra foi analisada em triplicata.

\section{Cálculo dos índices de retenção}

A função "PROCV" do Excel ${ }^{\circledR}$ procura um valor, neste caso, o tempo de retenção de um componente, por exemplo o pico 5 na figura 2 abaixo (coordenadas C9) em uma tabela com os dados série homóloga de $n$-alcanos (Material Suplementar, Planilha 4, matriz F9:F28) até encontrar um valor imediatamente menor ao procurado (Célula F12 da Planilha 2). Nesse ponto, a função retorna à planilha os dados das colunas correspondentes à linha na qual a busca parou ( $\mathrm{o} \mathrm{t}_{\mathrm{R}}$ do alcano em F12). A mesma função retorna também a constante "n" da fórmula para o cálculo do IRL (Planilha 2, célula G12). Para o $t_{R}$ do padrão que elui imediatamente após o pico de interesse (Célula F13), utilizou-se uma combinação das funções "ÍNDICE" e "CORRESP". ${ }^{19}$ Finalmente, o índice de retenção linear do pico é calculado utilizando a aproximação de Van den Dool e Kratz. ${ }^{7}$ Os algoritmos detalhados estão no Material Suplementar, bem como um arquivo com as planilhas.

Somente para fazer a interpolação manual dos tempos de retenção dos 40 ou 50 constituintes de uma amostra com os tempos de retenção da série homóloga são necessários cerca de 10 minutos ou pouco mais. Com a automatização desse procedimento, o tempo necessário é apenas aquele para colar os dados das amostras na Planilha 1 e os tempos de eluição dos componentes da série homóloga, na Planilha 2, sendo inferior a 30 segundos. Para a análise de diversas amostras utilizando-se a mesma injeção da série homóloga, a Planilha 2 precisa ser preenchida apenas uma vez e, ao se salvar o arquivo, os valores serão usados para todas as demais amostras que forem sendo analisadas. Em um laboratório como o da Embrapa Agroindústria de Alimentos, que processa até 1200 amostras por ano, sem contar as replicatas, isto equivale a uma economia de mão-de-obra de 200 h/ano. Mais ainda, com o processo automatizado, erros de digitação de valores ou de posicionamento nas tabelas são eliminados, minimizando erros de identificação, bem como o retrabalho.

\section{Análise Qualitativa}

Esta é, sem dúvida, a etapa crítica (e a etapa lenta, também) de todo o processo. Os resultados da busca em bases de espectros devem ser cuidadosamente comparados com o espectro de massas experimental. O mesmo vale para os índices de retenção. Foram usadas a base de dados da NIST ${ }^{18}$ e tanto o livro quanto a base de dados do prof. Adams ${ }^{9}$ para as identificações.

Variações de até 5 unidades no IRL são aceitáveis, porém diferenças maiores que 10 unidades são um forte indício de que a proposta de identificação não é a mais adequada. Frequentemente se observam espectros de massas virtualmente idênticos para substâncias diferentes, como isômeros de posição, e o uso criterioso dos IRL é essencial para uma identificação correta. A coinjeção de padrões autênticos, quando disponíveis, muito contribui para a identificação inequívoca.

Nos casos para os quais a diferença nos espectros de massas e nos índices de retenção não são suficientes para a correta identificação, 
Replicata 1

\begin{tabular}{crr} 
Massa 1: & $\mathbf{0 , 0 0 8 2} \mathbf{~ g}$ & \\
\hline Pico & \multicolumn{1}{c}{ Tr 1 } & Área 1 \\
\hline 1 & 4,44 & 32,2 \\
2 & 5,319 & 45,9 \\
3 & 5,404 & 569,1 \\
4 & 5,734 & 24,8 \\
5 & 6,438 & 3 \\
6 & 6,79 & 459,3 \\
7 & 7,699 & 5,2 \\
8 & 11,939 & 11,1 \\
9 & 18,122 & 17,9 \\
10 & 18,277 & 7 \\
11 & 18,62 & 7 \\
12 & 19,628 & 26,6 \\
13 & 19,73 & 18,4 \\
14 & 20,006 & 29,4 \\
15 & 20,224 & 24,1 \\
16 & 20,313 & 23,7 \\
17 & 20,729 & 13,8 \\
18 & 21,304 & 80,9 \\
19 & 21,697 & 7,4 \\
20 & 22,055 & 11,4 \\
21 & 22,622 & 14 \\
22 & 23,583 & 6,9 \\
23 & 23,717 & 245,9 \\
24 & 24,325 & 367,9 \\
25 & 24,502 & 9,4 \\
26 & 24,616 & 6,1 \\
27 & 25,384 & 20,3 \\
28 & 25,464 & 19,6 \\
29 & 25,568 & 24,2 \\
30 & 27,366 & 27,5 \\
& &
\end{tabular}

Replicata 2

Massa 2: 0,0082

\begin{tabular}{crr}
\hline Pico & \multicolumn{1}{c}{ Tr 2 } & Área 2 \\
\hline 1 & 4,453 & 54,3 \\
2 & 5,335 & 76,8 \\
3 & 5,425 & 953 \\
4 & 5,749 & 41,5 \\
5 & 6,454 & 4,9 \\
6 & 6,813 & 773,8 \\
7 & 7,716 & 8,6 \\
8 & 11,949 & 19 \\
9 & 18,142 & 9,6 \\
10 & 18,289 & 12,2 \\
11 & 18,633 & 11,9 \\
12 & 19,64 & 46,5 \\
13 & 19,742 & 32,3 \\
14 & 20,017 & 50,7 \\
15 & 20,235 & 42,7 \\
16 & 20,325 & 23 \\
17 & 20,741 & 21,6 \\
18 & 21,317 & 139,7 \\
19 & 21,708 & 11,5 \\
20 & 22,066 & 19,7 \\
21 & 22,634 & 23,9 \\
22 & 23,599 & 15,3 \\
23 & 23,737 & 442,4 \\
24 & 24,349 & 664 \\
25 & 24,513 & 16,5 \\
26 & 24,628 & 30,9 \\
27 & 25,393 & 34,4 \\
28 & 25,474 & 33,5 \\
29 & 25,577 & 41,8 \\
30 & 27,376 & 47,6
\end{tabular}

Replicata 3

\begin{tabular}{ccr} 
Massa 3: & $\mathbf{0 , 0 0 8 2} \mathbf{g}$ & \\
\hline Pico & Tr 3 & Área 3 \\
\hline 1 & 4,455 & 39,7 \\
2 & 5,336 & 52,7 \\
3 & 5,421 & 667 \\
4 & 5,752 & 27,4 \\
5 & 6,456 & 3,3 \\
6 & 6,808 & 515,1 \\
7 & 7,718 & 5,7 \\
8 & 11,959 & 11,3 \\
9 & 18,139 & 10,2 \\
10 & 18,292 & 7,4 \\
11 & 18,635 & 7,1 \\
12 & 19,64 & 27,7 \\
13 & 19,742 & 19,4 \\
14 & 20,018 & 30,5 \\
15 & 20,235 & 25,5 \\
16 & 20,326 & 18,5 \\
17 & 20,741 & 13 \\
18 & 21,316 & 85,7 \\
19 & 21,709 & 7,3 \\
20 & 22,066 & 12,1 \\
21 & 22,634 & 14,9 \\
22 & 23,594 & 7,2 \\
23 & 23,728 & 273,2 \\
24 & 24,337 & 416,2 \\
25 & 24,514 & 9,7 \\
26 & 24,627 & 12,4 \\
27 & 25,393 & 22,1 \\
28 & 25,475 & 21,7 \\
29 & 25,577 & 27,2 \\
30 & 27,377 & 31,8
\end{tabular}

Figura 1. Entrada de dados (Planilha 1)

ou não estão disponíveis na literatura, deve-se isolar e caracterizar o constituinte que se quer identificar, ou se registrá-lo como "não identificado" (n.i.)

No exemplo (figura 3), as colunas B, C e D são automaticamente importadas das planilhas anteriores. O responsável pela análise irá digitar os nomes dos constituintes identificados, incluindo o índice retenção da literatura. Na coluna $\mathrm{G}$ é informada a função química à qual pertence cada constituinte identificado. Note-se que, mesmo que o constituinte não seja identificado (linha 18, pico 10 na figura 3), é possível se determinar a qual classe química o componente pertente e, desse modo, efetuar a correção de sua área e os demais cálculos pertinentes. No exemplo mencionado, foi possível constatar que se trata efetivamente de um sesquiterpeno. Uma lista sugestiva encontra-se também na planilha 5, sob o título "Funções" (figura 4). A lista pode ser aumentada ou reduzida segundo a diversidade e natureza química dos constituintes a serem analisados. Nesse exemplo, álcoois, cetonas, éteres e óxidos monoterpênicos, embora discriminados na tabela "Funções", usam o mesmo fator de resposta, que foi calculado a partir da média dos fatores de resposta individuais do linalol, mentol, carvona, nerol e óxido de linalol, padrões disponíveis no laboratório no momento do estudo. Outra alternativa seria calcular os fatores separadamente, lançando os valores individuais na tabela.

A coluna $\mathrm{H}$ contém informação sobre a classe química na forma mais comum encontrada em artigos de óleos essenciais, ou seja, monoterpenos (MT), monoterpenos oxigenados (MO), sesquiterpenos (ST), sesquiterpenos oxigenados (SO) e outros (OT). Esses dados são usados depois para o cálculo do total percentual de cada classe presente. O preenchimento desta coluna é opcional, e não interfere com a quantificação dos constituintes.

Na coluna I são recuperados os fatores de resposta obtidos experimentalmente no equipamento usado para as análises. $\mathrm{Na}$ análise do óleo essencial de Lippia alba amostra RFV 2556, os fatores de resposta foram calculados pela média dos resultados das cinco concentrações injetadas de cada padrão. Em alguns casos, como para os sesquiterpenos, só se dispunha de um único padrão. Em outros (monoterpenoides oxigenados), cinco padrões foram usados e utilizou-se a média dos resultados.

Esses fatores foram então inseridos na Planilha 5, em tabela própria (Funções), mostrada na Figura 4.

\section{Fator de resposta relativo}

O fator de resposta relativo foi calculado na planilha 6 , a partir da informação dos fatores de resposta dos padrões injetados em relação ao fator de resposta do padrão interno. É interessante inserir o fator de resposta absoluto de cada componente primeiro e só depois calcular o fator de resposta relativo (FRR), pois diferentes análises no mesmo equipamento podem ser feitas com diferentes padrões internos. Vale 
=PROCV(C9;'2 Dados da Série Homóloga'!\$F9:'2 Dados da Série Homóloga'!\$G\$28;2;1)

=PROCV(C9;'2 Dados da Série Homóloga'!\$F9:'2 Dados da Série Homóloga'!\$G\$28;1;1)

\begin{tabular}{|c|c|c|c|c|c|c|}
\hline & B & C & D & E & F & G \\
\hline $\mathbf{8}$ & Pico & Tr & n & Tr Cn & Tr Cn+1 & IRL \\
\hline $\mathbf{9}$ & 5 & 6,790 & 10 & 5.963 & $9,11,5$ & 1026 \\
\hline $\mathbf{1 0}$ & 6 & 7,699 & 10 & 5,963 & 9,115 & 1055 \\
\hline $\mathbf{1 1}$ & 7 & 11,939 & 11 & 9,115 & 12,879 & 1175 \\
\hline
\end{tabular}

=ÍNDICE('2 Dados da Série Homóloga'!\$F\$9:\$F 28;CORRESP('2 Dados da Série Homóloga'!C9;'2 Dados da Série Homóloga'!\$F\$9:\$F\$28;1)+1;1)

Série de alcanos

\begin{tabular}{|c|c|c|}
\hline & F & G \\
\hline $\mathbf{8}$ & Tr Cn & $\mathbf{n}$ \\
\hline $\mathbf{9}$ & - & 7 \\
\hline $\mathbf{1 0}$ & - & 8 \\
\hline $\mathbf{1 1}$ & 3,74 & 9 \\
\hline $\mathbf{1 2}$ & 5,963 & 10 \\
\hline $\mathbf{1 3}$ & 9,115 & 11 \\
\hline $\mathbf{1 4}$ & 12,879 & 12 \\
\hline $\mathbf{1 5}$ & 16,888 & 13 \\
\hline $\mathbf{1 6}$ & 20,889 & 14 \\
\hline $\mathbf{1 7}$ & 24,784 & 15 \\
\hline $\mathbf{1 8}$ & 28,530 & 16 \\
\hline
\end{tabular}

Figura 2. Partes das Planilhas 4 (acima) e 2 (abaixo)

lembrar que o fator de resposta é função da natureza química, das condições de operação e das características físicas do detector. Uma vez calculados, se as condições de análise forem mantidas (temperatura do detector, vazão dos gases da chama), o fator pode ser usado durante um determinado período de tempo, em geral de seis a doze meses, período esse que vai variar conforme o uso do detector. Esse intervalo para um novo cálculo deve ser determinado empiricamente em cada equipamento, segundo o sistema de qualidade implantado no laboratório.

A condição ideal para o cálculo dos fatores de resposta é a injeção de padrões autênticos de cada analito a ser quantificado. Para o estudo de óleos essenciais, entretanto, esta condição não pode ser cumprida: não há padrões comerciais para a maioria dos constituintes identificados em OE. Uma das formas de se contornar esse problema é o uso de padrões da mesma classe química. A diferença nos fatores de resposta entre o padrão usado e um analito da mesma classe é, em geral, menor que $0,05,{ }^{22}$ valor que não irá impactar expressivamente a quantificação.

É interessante observar que o uso de fatores de resposta, embora praticamente não provoque alteração nas áreas de algumas classes de constituintes, como mono e sesquiterpenos (Tabela 1), e, desse modo, não altere sua a quantificação, leva a uma grande variação em outros casos (monoterpenoides oxigenados), onde a diferença e, logo, o erro na medida, chega a $50 \%$ (FRR médio de 1,48). Portanto, para a quantificação acurada faz-se necessária utilização dos fatores de resposta.
Quando se utiliza um padrão interno da mesma classe química dos constituintes a serem analisados, a diferença entre os fatores de resposta é minimizada, o que faz com que o fator de resposta relativo seja próximo à unidade. Esta pode ser uma alternativa para a análise de uma amostra com apenas uma classe de substâncias. Para óleos essenciais e extratos voláteis, entretanto, este é raramente o caso, dada a diversidade de classes químicas observadas neste tipo de amostra. O mesmo procedimento de cálculo foi utilizado para a análise da fração volátil de Temnadenia odorifer ${ }^{20}$ e do óleo essencial de Bidens graveolens. ${ }^{21}$

Uma alternativa para ser aplicada quando padrões autênticos não estão disponíveis é o uso de fatores de resposta preditivos ou teóricos..$^{23,24}$ Cachet e colaboradores publicaram uma planilha de cálculos em Excel que pode ser inserida no conjunto de planilhas proposto aqui, substituindo a planilha de cálculo de fatores de resposta. ${ }^{24}$ Nesse caso, o padrão interno tem que ser o especificado no artigo (octanoato de metila), uma vez que a fórmula proposta foi ajustada para o uso dessa substância como padrão. Os erros comparativos entre o cálculo de fatores de resposta teóricos e sua determinação experimental, para uma série de padrões testados, foi inferior a $5 \% .^{24}$

\section{Análise quantitativa}

Os dados de área absoluta obtidos do DIC foram transferidos diretamente para a Planilha 1, como mostrado anteriormente. Para um 


\begin{tabular}{|c|c|c|c|c|c|c|c|c|}
\hline & B & C & D & $\mathbf{E}$ & $\mathbf{F}$ & G & H & 1 \\
\hline 8 & Pico & $\operatorname{Tr}$ & $\mathrm{IRL}_{\text {calc }}$ & IRL $_{\text {lit }}$ & Identificação & Função & Classe & FR \\
\hline 9 & 1 & 4,440 & 931 & 932 & alfa-pineno & 1 & MT & 1,05 \\
\hline 10 & 2 & 5,319 & 971 & 969 & sabineno & 1 & MT & 1,05 \\
\hline 11 & 3 & 5,404 & 975 & 974 & beta-pineno & 1 & MT & 1,05 \\
\hline 12 & 4 & 5,734 & 990 & 988 & mirceno & 1 & MT & 1,05 \\
\hline 13 & 5 & 6,438 & 1015 & 1014 & alfa-terpineno & 1 & MT & 1,05 \\
\hline 14 & 6 & 6,790 & 1026 & 1025 & limoneno + beta-felandreno & 1 & MT & 1,05 \\
\hline 15 & 7 & 7,699 & 1055 & 1054 & gama-terpineno & 1 & MT & 1,05 \\
\hline 16 & 8 & 11,939 & 1175 & 1174 & terpinen-4-ol & 3 & $\mathrm{MO}$ & 1,48 \\
\hline 17 & 9 & 18,122 & 1331 & 1338 & bicicloelemeno & 9 & ST & 1,01 \\
\hline 18 & 10 & 18,277 & 1335 & - & n.i. & 9 & ST & 1,01 \\
\hline 19 & 11 & 18,620 & 1343 & 1345 & alfa-cubebeno & 9 & ST & 1,01 \\
\hline 20 & 12 & 19,628 & 1368 & 1369 & ciclosativeno & 9 & ST & 1,01 \\
\hline 21 & 13 & 19,730 & 1371 & 1374 & alfa-copaeno & 9 & ST & 1,01 \\
\hline 22 & 14 & 20,006 & 1378 & 1387 & alfa-isocomeno & 9 & ST & 1,01 \\
\hline 23 & 15 & 20,224 & 1383 & 1387 & beta-cubebeno & 9 & ST & 1,01 \\
\hline 24 & 16 & 20,313 & 1386 & 1389 & beta-elemeno & 9 & ST & 1,01 \\
\hline 25 & 17 & 20,729 & 1396 & 1407 & beta-isocomeno & 9 & ST & 1,01 \\
\hline 26 & 18 & 21,304 & 1411 & 1417 & (E)-beta-cariofileno & 9 & ST & 1,01 \\
\hline 27 & 19 & 21,697 & 1421 & 1430 & beta-copaeno & 9 & ST & 1,01 \\
\hline 28 & 20 & 22,055 & 1430 & 1439 & aromadendreno & 9 & ST & 1,01 \\
\hline 29 & 21 & 22,622 & 1444 & 1452 & alfa-humuleno & 9 & ST & 1,01 \\
\hline 30 & 22 & 23,583 & 1469 & 1478 & gama-muuroleno & 9 & ST & 1,01 \\
\hline 31 & 23 & 23,717 & 1473 & 1480 & germacreno D & 9 & ST & 1,01 \\
\hline 32 & 24 & 24,325 & 1488 & 1494 & biciclogermacreno & 9 & ST & 1,01 \\
\hline 33 & 25 & 24,502 & 1493 & 1500 & alfa-muroleno & 9 & ST & 1,01 \\
\hline 34 & 26 & 24,616 & 1496 & 1500 & alfa-muroleno & 9 & ST & 1,01 \\
\hline 35 & 27 & 25,384 & 1516 & 1522 & delta-cadineno & 9 & ST & 1,01 \\
\hline 36 & 28 & 25,464 & 1518 & 1529 & kesane & 12 & so & 1,35 \\
\hline 37 & 29 & 25,568 & 1521 & 1534 & liguloxido & 12 & SO & 1,35 \\
\hline 38 & 30 & 27,366 & 1569 & 1577 & espatulenol & 11 & so & 1,35 \\
\hline
\end{tabular}

Figura 3. Planilha 5 - Análise Qualitativa

\begin{tabular}{lcc}
\hline Funções & Código & FR \\
\hline Monoterpenos & 1 & 1,02 \\
Monoterpenos aromáticos & 2 & - \\
Álcoois monoterpênicos & 3 & 1,44 \\
Cetonas monoterpênicas & 4 & 1,44 \\
Éteres monoterpênicos & 5 & 1,44 \\
Ésteres monoterpênicos & 6 & 1,21 \\
Epóxidos monoterpênicos & 7 & 1,44 \\
Fenóis monoterpênicos & 8 & - \\
Sesquiterpenos & 9 & 0,98 \\
Sesquiterpenos aromáticos & 10 & 0,98 \\
Álcoois sesquiterpênicos & 11 & 1,31 \\
Cetonas sesquiterpênicas & 12 & 1,31 \\
Éteres sesquiterpênicos & 13 & 1,31 \\
Ésteres sesquiterpênicos & 14 & 1,31 \\
Epóxidos sesquiterpênicos & 15 & 1,31 \\
\hline
\end{tabular}

Figura 4. Planilha 5 - Tabela de funções e fatores de resposta (FR) estudo em triplicata, os dados de cada replicata devem ser transferidos para as colunas D, $\mathrm{H} \mathrm{e} \mathrm{L} \mathrm{desta} \mathrm{planilha} \mathrm{(figura} \mathrm{1).} \mathrm{É} \mathrm{importante}$ informar a massa de amostra pesada para o preparo da solução de análise, nas células C7, G7 e K7. No exemplo, como a mesma solução foi injetada em triplicata, a massa foi a mesma.

Diversas formas de expressar o resultado podem ser calculadas e a planilha de análise quantitativa (Planilha 7), por comodidade, inclui essas diferentes apresentações (Figura 5). Utilizando-se a massa do padrão interno (ou sua concentração) e os fatores de resposta, calculados ou estimados, o resultado final da análise pode ser expresso de forma relativa (percentual) ou absoluta. A primeira é mais comum nos resultados de análise do perfil de OE; a segunda, nas análises de contaminantes e componentes específicos (target analysis). As fórmulas podem ser ajustadas para exibição do resultado com diferentes unidades, facilitando a comparação com dados da literatura.

Todos os valores desta planilha são automaticamente recuperados e/ou calculados a partir das planilhas precedentes. A partir daí as planilhas seguintes do conjunto (Planilhas 8, 9,10 e 11) também são preenchidas automaticamente, e o usuário irá selecionar a planilha com a 


\begin{tabular}{|c|c|c|c|c|c|c|c|c|c|c|}
\hline B & C & D & E & $\mathbf{F}$ & G & H & I & J & K & $\mathbf{L}$ \\
\hline Pico & $\operatorname{Tr} 1$ & Área 1 & FRR & A 1 norm & Área 1 cor & A 1 cor nor & A1\% & A1 norm \% & A1 norm cor $\%$ & Absoluta 1 \\
\hline 1 & 4,440 & 32,200 & 1,047 & 0,516 & 33,721 & 0,540 & 1,491 & 1,491 & 1,499 & 5857,092 \\
\hline 2 & 5,319 & 45,900 & 1,047 & 0,736 & 48,068 & 0,770 & 2,125 & 2,125 & 2,136 & 8349,084 \\
\hline 3 & 5,404 & 569,100 & 1,047 & 9,120 & 595,977 & 9,551 & 26,347 & 26,347 & 26,490 & 103517,729 \\
\hline 4 & 5,734 & 24,800 & 1,047 & 0,397 & 25,971 & 0,416 & 1,148 & 1,148 & 1,154 & 4511,052 \\
\hline 5 & 6,438 & 3,000 & 1,047 & 0,048 & 3,142 & 0,050 & 0,139 & 0,139 & 0,140 & 545,692 \\
\hline 6 & 6,790 & 459,300 & 1,047 & 7,361 & 480,992 & 7,708 & 21,264 & 21,264 & 21,379 & 83545,410 \\
\hline 7 & 7,699 & 5,200 & 1,047 & 0,083 & 5,446 & 0,087 & 0,241 & 0,241 & 0,242 & 945,866 \\
\hline 8 & 11,939 & 11,100 & 1,478 & 0,178 & 16,411 & 0,263 & 0,514 & 0,514 & 0,729 & 2850,437 \\
\hline 9 & 18,122 & 17,900 & 1,006 & 0,287 & 18,010 & 0,289 & 0,829 & 0,829 & 0,801 & 3128,276 \\
\hline 10 & 18,277 & 7,000 & 1,006 & 0,112 & 7,043 & 0,113 & 0,324 & 0,324 & 0,313 & 1223,348 \\
\hline 11 & 18,620 & 7,000 & 1,006 & 0,112 & 7,043 & 0,113 & 0,324 & 0,324 & 0,313 & 1223,348 \\
\hline 12 & 19,628 & 26,600 & 1,006 & 0,426 & 26,764 & 0,429 & 1,231 & 1,231 & 1,190 & 4648,723 \\
\hline 13 & 19,730 & 18,400 & 1,006 & 0,295 & 18,513 & 0,297 & 0,852 & 0,852 & 0,823 & 3215,658 \\
\hline 14 & 20,006 & 29,400 & 1,006 & 0,471 & 29,581 & 0,474 & 1,361 & 1,361 & 1,315 & 5138,063 \\
\hline 15 & 20,224 & 24,100 & 1,006 & 0,386 & 24,248 & 0,389 & 1,116 & 1,116 & 1,078 & 4211,813 \\
\hline 16 & 20,313 & 23,700 & 1,006 & 0,380 & 23,846 & 0,382 & 1,097 & 1,097 & 1,060 & 4141,908 \\
\hline 17 & 20,729 & 13,800 & 1,006 & 0,221 & 13,885 & 0,223 & 0,639 & 0,639 & 0,617 & 2411,744 \\
\hline 18 & 21,304 & 80,900 & 1,006 & 1,296 & 81,398 & 1,304 & 3,745 & 3,745 & 3,618 & 14138,410 \\
\hline 19 & 21,697 & 7,400 & 1,006 & 0,119 & 7,446 & 0,119 & 0,343 & 0,343 & 0,331 & 1293,254 \\
\hline 20 & 22,055 & 11,400 & 1,006 & 0,183 & 11,470 & 0,184 & 0,528 & 0,528 & 0,510 & 1992,310 \\
\hline 21 & 22,622 & 14,000 & 1,006 & 0,224 & 14,086 & 0,226 & 0,648 & 0,648 & 0,626 & 2446,696 \\
\hline 22 & 23,583 & 6,900 & 1,006 & 0,111 & 6,943 & 0,111 & 0,319 & 0,319 & 0,309 & 1205,872 \\
\hline 23 & 23,717 & 245,900 & 1,006 & 3,941 & 247,415 & 3,965 & 11,384 & 11,384 & 10,997 & 42974,475 \\
\hline 24 & 24,325 & 367,900 & 1,006 & 5,896 & 370,166 & 5,932 & 17,032 & 17,032 & 16,453 & 64295,687 \\
\hline 25 & 24,502 & 9,400 & 1,006 & 0,151 & 9,458 & 0,152 & 0,435 & 0,435 & 0,420 & 1642,782 \\
\hline 26 & 24,616 & 6,100 & 1,006 & 0,098 & 6,138 & 0,098 & 0,282 & 0,282 & 0,273 & 1066,061 \\
\hline 27 & 25,384 & 20,300 & 1,006 & 0,325 & 20,425 & 0,327 & 0,940 & 0,940 & 0,908 & 3547,710 \\
\hline 28 & 25,464 & 19,600 & 1,350 & 0,314 & 26,460 & 0,424 & 0,907 & 0,907 & 1,176 & 4595,944 \\
\hline 29 & 25,568 & 24,200 & 1,350 & 0,388 & 32,670 & 0,524 & 1,120 & 1,120 & 1,452 & 5674,585 \\
\hline 30 & 27,366 & 27,500 & 1,350 & 0,441 & 37,125 & 0,595 & 1,273 & 1,273 & 1,650 & 6448,391 \\
\hline
\end{tabular}

Figura 5. Planilha 7-Quantitativa (parte)

forma de apresentação de resultados que lhe for mais conveniente.

Para a análise de óleos essenciais e de constituintes de frações voláteis, a recomendação da IOFI (International Organization of the Flavor Industry) é que se use preferencialmente a quantificação absoluta ou, no mínimo, a quantificação por área normalizada e corrigida. ${ }^{24}$ Esses valores correspondem aos dados das colunas L e K da Planilha 7, respectivamente. Quando não se dispuser de padrões para o cálculo dos fatores de resposta, fatores teóricos (preditivos) devem ser usados. ${ }^{24}$

\section{CONCLUSÃO}

Com a utilização do conjunto de planilhas há redução no tempo necessário para o tratamento dos dados cromatográficos. Os resultados são apresentados de forma mais organizada e, com a redução da necessidade de se copiar dados, os erros de transcrição são também reduzidos. As planilhas são facilmente editáveis e a elas podem ser incorporadas informações adicionais das amostras, logotipos e símbolos, fórmulas químicas e outros detalhamentos.

\section{MATERIAL SUPLEMENTAR}

O conjunto de planilhas para cálculo quantitavo e qualitativo utiliza neste trabalho está disponível em http://quimicanova.sbq.org. br, na forma de arquivo Excel $^{\circledR}$, com acesso livre.

\section{AGRADECIMENTOS}

E.G.B. e H.R.B. agradecem ao $\mathrm{CNPq}$ por concessão de bolsas PIBIC e PQ, respectivamente.

\section{REFERÊNCIAS}

1. International Organization for Standardization. ISO 9235:2013. Aromatic natural raw materials - Vocabulary. Genebra, 2013 35p.

2. Bizzo, H. R.; Lopes, D.; Abdala, R.V. Pimentel, F. A.; Souza, J. A., Pereira, M. V. G.; Bergter, L.; Guimarães, E. F.; Flavour Frag. J. 2001, 16, 113.

3. Belhassen, E.; Baldovini, N.; Brevard, H.; Meierhenrich, U. J.; Filippi, J. J.; Chem. Biodivers. 2014, 11, 1821.

4. Lamparsky, D. Em Capillary Gas Chromatography in Essential oil Analysis; Sandra, P.; Bicchi, C. eds.; Alfred Huethig Verlag: Heidelberg, 1987, cap.6.

5. Bicchi, C.; Liberto, E.; Matteodo, M.; Sgorbini, B.; Mondello, L.; Zellner, B. A.; Costa, R.; Rubiolo, P.; Flavour Fragr. J. 2008, 23, 382.

6. Kováts, E.; Helv. Chim. Acta 1958, 41, 1915.

7. Van Den Dool, H.; Kratz, P.D.; J. Chromatogr. 1963, 11, 463.

8. Zellner, B. A.; Bicchi, C.; Dugo, P.; Rubiolo, P.; Dugo, G.; Mondello, L.; Flavour Fragr. J. 2008, 23, 297.

9. Adams, R. P.; Identification of essential oil components by gas chromatography / mass spectrometry, $4^{\text {th }}$ ed., Carol Stream: Allured Publishing Corporation, 2007. 
10. https://webbook.nist.gov/chemistry/gc-ri/, acessada em Janeiro 2019.

11. http://www.flavornet.org, acessada em Agosto 2019.

12. http://www.pherobase.com, acessada em Agosto 2019.

13. Agilent; GC ChemStation B.04.02; Agilent Technologies, Palo Alto (EUA), 2009.

14. Alencar, J. W.; Craveiro, A. A.; Matos, F. J. A.; J. Nat. Prod. 1984, 47, 890.

15. McLafferty, F.; Wiley Registry of Mass Spectral Data, 11th ed., New York: Wiley Interscience, 2016; Mondello, L.; Mass Spectra of Flavors and Fragrances of Natural and Synthetic Compounds, $3^{\text {rd }}$ ed.; New York: Wiley-Blackwell, 2015.

16. http://www.leffingwell.com/baciseso.htm, acessada em Janeiro 2019.

17. https://www.gcimage.com/wiley.html, acessada em Janeiro 2019.

18. NIST Mass Spectral Library, v11, National Institute of Standards and Technology, Gaithersburg, 2011.

19. Gama, P. E.; Barboza, E. G.; Santos, M. C. S.; Bizzo, H. R. Automação de Planilhas Eletrônicas Para o Cálculo de Índices de Retenção na Análise de Óleos Essenciais. Rio de Janeiro: Embrapa Agroindústria de Alimentos, 2017. 7 p.
20. Silva, R. F.; Tinoco, N. A. B.; Tsukui, A.; Koschnitzke, C.; Silva-Batista, I. C.; Rezende, C. M.; Bizzo, H. R.; J. Braz. Chem. Soc. 2019, 30, 388.

21. Silva, A. C. R.; Bizzo, H. R.; Vieira, R. F.; Bringel Junior, J. A.; Azevedo, D. A.; Uekane, T. M.; Rezende, C. M.; Flavour Fragr. J. 2020, 35,79 .

22. Costa, R.; Zellner, B. A.; Crupi, M. L.; Fina, M. R.; Valentino, M. R.; Dugo, P.; Dugo, G.; Mondello, L.; Flavour Fragrance J. 2008, 23, 40.

23. Saint-Laumer, J. Y. ; Cicchetti, E.; Merle, P.; Egger, J.; Chaintreau, A.; Anal. Chem. 2010, 82, 6457.

24. Cachet, T.; Brevard, H.; Chaintreau, A.; Demyttenaere, J.; French, L.; Gassenmeier, K.; Joulain, D.; Koenig, T.; Leijs, H.; Liddle, P.; Loesing, G.; Marchant, M.; Merle, Ph.; Saito, K.; Schippa, C.; Sekiya F.; Smith, T.; Flavour Fragr. J. 2016, 31, 191. 